\title{
NO-NEEDLE ONE-QUADRANT SUB-TENON ANAESTHESIA FOR PANRETINAL PHOTOCOAGULATION
}

\author{
J. D. STEVENS, A. J. E. FOSS and A. M. P. HAMILTON \\ London
}

\begin{abstract}
SUMMARY
Panretinal photocoagulation (PRP) is tolerated well by most patients using topical anaesthesia alone, though there are a significant number of patients who experience pain. Additional local anaesthesia alternatives for these patients include retrobulbar, peribulbar or subconjunctival injection. Deep introduction of a sharp needle may rarely cause damage to orbital structures, whereas noneedle sub-Tenon irrigation of local anaesthetic solution to the posterior Tenon's space theoretically avoids these risks. A one-quadrant, inferior-nasal, sub-Tenon delivery of $1.5-2 \mathrm{ml}$ plain $2 \%$ lignocaine was administered and PRP performed on 12 eyes of 12 patients who were previously intolerant of PRP by topical anaesthesia alone. To assess the efficacy of anaesthesia, patients were asked to score pain, using a visual analogue score chart graded from 0 to 10. If patients were unable to see the chart, or read the accompanying text, a verbal explanation and description of the scoring chart was performed. Where PRP was performed with topical amethocaine $1 \%$ alone, pain scores were graded as median 8 , mean 8.5 and range 6-10. The administration of sub-Tenon anaesthesia was well tolerated with a median pain score of 1.5 , mean 1.9 and range 0-5. PRP after sub-Tenon administration was successfully completed in 11 of the 12 patients with a median pain score of 1.5 , mean 1.8 and range of $0-9$. The range was wide due to one patient with a high pain score who was intolerant of PRP in spite of the sub-Tenon delivery. Assessment of pain scores demonstrates significantly better anaesthesia with sub-Tenon irrigation than topical treatment alone $(p<0.005$, Wilcoxon ranked sign test). If a patient tolerates PRP with simple topical anaesthesia then this is the method of choice. Topical anaesthesia is non-invasive, though if pain is experienced, one-quadrant sub-Tenon anaesthesia can provide effective anaesthesia for PRP with the benefit of seeking to avoid the risks of sharp needle techniques.
\end{abstract}

Panretinal photocoagulation (PRP) has greatly improved the prognosis for patients with proliferative diabetic retinopathy. While most patients tolerate PRP well under

Correspondence to: J. D. Stevens, Moorfields Eye Hospital, City Road, London EC1V 2PD, UK. topical anaesthesia alone, there are a significant number of patients who experience discomfort and pain. The alternative for these patients involves either retrobulbar, peribulbar or subconjunctival injection, or general anaesthesia. The local anaesthetic techniques use a sharp, or relatively sharp, needle which for the retrobulbar and peribulbar techniques is 'blindly' placed into the orbit to deliver the anaesthetic solution. Sharp needle sub-Tenon injection $10 \mathrm{~mm}$ posterior to the limbus in the quadrant for photocoagulation has been reported as providing good anaesthesia for PRP. ${ }^{1}$ It is useful if sector photocoagulation is required, but would involve multiple quadrant delivery if PRP is to be performed.

Deep introduction of a sharp needle may rarely cause damage to orbital structures, including perforation of the globe, ${ }^{2}$ a blood vessel or the optic nerve sheath, and anaesthetic may perfuse into the subarachnoid space causing respiratory or cardiovascular arrest. ${ }^{3}$ For these reasons, sharp needle local anaesthetic blocks should be administered where full resuscitation facilities are available.

Friedberg and Palmer ${ }^{4}$ reported a single-quadrant, subTenon anaesthetic technique, involving irrigation of local anaesthetic solution to the superior-nasal quadrant of posterior Tenon's space. They performed PRP to 40 eyes and questioned patients regarding pain but did not perform visual analogue pain scoring. Friedberg and Palmer reported access to the superior-nasal quadrant as being difficult in anxious patients due to Bell's phenomenon, and in these patients the inferior-nasal quadrant was chosen. Another technique of sub-Tenon delivery which has been shown to be well tolerated when used for cataract extraction specifically uses the inferior-nasal quadrant. ${ }^{5}$ This sub-Tenon technique is similar to that of Friedberg and Palmer ${ }^{4}$ but differs in not involving a subconjunctival injection using a sharp needle and also delivery of anaesthetic is to the inferior-nasal quadrant in all patients. Use of sub-Tenon delivery with a low volume $(<3 \mathrm{ml})$ avoids complications associated with a large volume of local anaesthetic agent such as a tight orbit, ${ }^{6}$ raised intraocular pressure $^{7}$ and systemic absorption with possible toxicity.

We describe the use and evaluation of patient tolerance 
of this infero-nasal one-quadrant technique for PRP for treatment of proliferative diabetic retinopathy in an outpatient setting.

\section{MATERIALS AND METHODS}

Twelve patients ( 4 men and 8 women) aged from 18 years to 56 years with proliferative diabetic retinopathy who were to receive PRP had a local anaesthetic block by onequadrant sub-Tenon irrigation. All had expressed anxiety about discomfort experienced during a previous treatment episode. Each had received previous PRP using topical anaesthesia of amethocaine $1 \%$. Further treatment was necessary, all patients having active new vessels on fundal examination.

\section{One-Quadrant Sub-Tenon Technique}

After informed consent, the pupil is dilated using four drops of cyclopentolate $1 \%$ and prophylactic chloramphenicol $0.5 \%$ is applied to the inferior conjunctival fornix. Two drops of amethocaine $1 \%$ are administered to the inferior conjunctival fornix. The one-quadrant sub-Tenon technique used has been previously published ${ }^{5}$ and the method used for PRP is summarised below.

The patient lies down and is asked to look upward and outward with respect to the eye to be anaesthetised, exposing the infero-temporal bulbar conjunctiva without the need for a lid speculum. A small fold of bulbar conjunctiva is grasped with Moorfields forceps or similar, and a 'nick' incision made in the conjunctiva using blunttipped Westcott's spring scissors. A new blunt-tipped, purpose-designed, curved 19-swg irrigating cannula ${ }^{8}$ is then used to deliver a small quantity of local anaesthetic just sufficient to raise a small bleb of conjunctiva. This elevation of conjunctiva facilitates identification of Tenon's fascia, which appears as opaque and whitish tissue. The Westcott scissors are then reintroduced through the previous incision in the conjunctiva and used to create another 'nick' in Tenon's fascia. Moorfields forceps are used to grip the conjunctival edge where the small nick was made and the blunt curved cannula is then inserted onto bare sclera and glided along a path following the contour of the globe, until just posterior to the equator. It is very important to pass through conjunctiva and Tenon's fascia to reach bare sclera, and therefore into Tenon's space, before sliding the cannula posteriorly. Gentle pressure is applied to the syringe and after a few seconds a slight 'give' is felt, solution begins to flow and the cannula may be further advanced to a position immediately posterior to the equator of the globe. After delivery of approximately $1.5-2 \mathrm{ml}$ of plain lignocaine $2 \%$ anaesthetic solution, the cannula is withdrawn. At least 5 minutes is allowed for diffusion to occur before commencing treatment. After PRP treatment is completed, a course of topical chloramphenicol four times daily for 1 week is prescribed. This is to provide prophylactic antibiotic cover for the incision to conjunctiva and Tenon's fascia.

\section{Panretinal Photocoagulation}

Laser application was with a Coherent argon laser, using argon green, $515 \mathrm{~nm}$ wavelength and applied using a Volk TransEquator ${ }^{9}$ fundus contact lens. A constant spot size of $500 \mu \mathrm{m}$ was used throughout. The laser spot duration time was $0.1-0.15$ seconds, with the exception of one patient who had treatment of 0.4 seconds duration. This was due to the presence of a dense vitreous haemorrhage requiring increased energy delivery. The energy used was 280$1000 \mathrm{~mW}$ (mean $529 \mathrm{~mW}$ ).

\section{Pain Score Assessment}

Each patient was shown a $10 \mathrm{~cm}$, visual analogue pain score chart with numerical and descriptive rating scale, graded from 0 to $10 .^{5}$ Patients were shown the chart, by

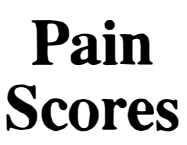

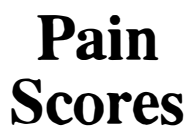

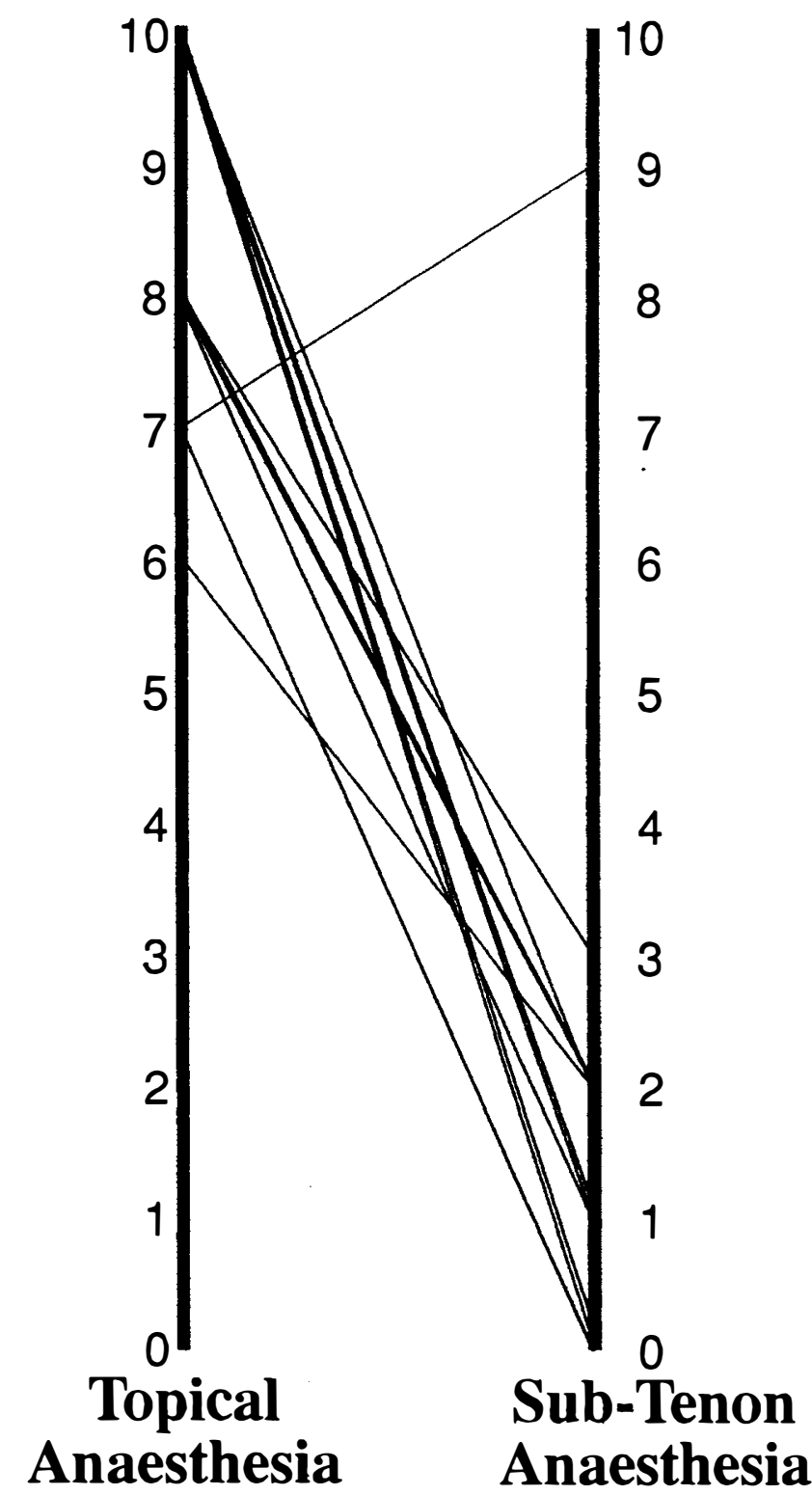

Fig. 1. Pain scores for PRP after topical and sub-Tenon anaesthesia. Pain scores for sub-Tenon anaesthesia were low except for patient 7. Pain scores for topical anaesthesia from memory recall were high. 
one investigator only, after completion of the PRP treatment and invited to score where on the chart they would grade any discomfort or pain experienced. If unable to see the chart, or read the accompanying text (printed at N10 size), a verbal explanation and description of the scoring chart was performed.

\section{RESULTS}

The results of the patient pain scores are presented graphically in Fig. 1. The pain experienced during the previous treatment episode when PRP was performed with topical amethocaine $1 \%$ alone, was graded as median 8 , mean 8.5 with a range of $6-10$. The administration of the sub-Tenon anaesthesia was well tolerated with a median pain score of 1.5 , mean 1.9 and range $0-5$.

PRP after sub-Tenon administration was tolerated by 11 of the 12 patients with a median of 1.5 , mean 1.8 and range of $0-9$. The range was wide due to one patient with a high pain score who was intolerant of PRP in spite of the subTenon delivery.

Sub-Tenon irrigation allowed 11 of 12 previously intolerant patients to complete a PRP treatment episode. Comparison of pain scores demonstrates significantly better anaesthesia with sub-Tenon irrigation than topical treatment alone $(p<0.005$, Wilcoxon ranked sign test). Fig. 2 plots the PRP pain scores for each of the 12 patients for topical anaesthesia and after sub-Tenon anaesthesia. All but one patient scored sub-Tenon anaesthesia as 0-3 on the chart, indicating that PRP is well tolerated by these patients. One patient (no. 7) scored 2 for the delivery of the sub-Tenon anaesthesia and 9 for the delivery of the
PRP. The sub-Tenon delivery appeared to have no anaesthetic effect in this instance. This patient requested an alternative form of anaesthesia for future treatment episodes; the remaining 11 patients indicated that they would prefer sub-Tenon anaesthesia to topical anaesthesia if future PRP were required.

All 12 patients developed a subconjunctival haemorrhage occupying one or two quadrants of the bulbar conjunctiva and mild chemosis in the infero-nasal quadrant. Two patients developed marked ocular pain with nausea and vomiting 4 hours after administration of the anaesthetic and laser treatment which settled over the course of the same day. This coincided with the recovery of ocular sensation as the effect of the plain lignocaine $2 \%$ subsided. Neither patient had a rise in intraocular pressure.

Patient no. 7 was the one patient who experienced significant pain and had only 293 burns before PRP treatment was terminated. Patient no. 3 received only 361 burns due to a poor view from vitreous haemorrhage. The other 10 patients received an average of 690 burns (range 480-1204).

\section{DISCUSSION}

Possible sources of bias in the study design are that the patients offered sub-Tenon anaesthesia are a small group ( $n=12$ ) selected to be intolerant of PRP under topical anaesthesia and that the pain of PRP performed at the treatment session was compared with that of a previous session from long-term memory. The patient group may also have a different response to pain from PRP delivery or be less tolerant of a painful stimulus than an otherwise

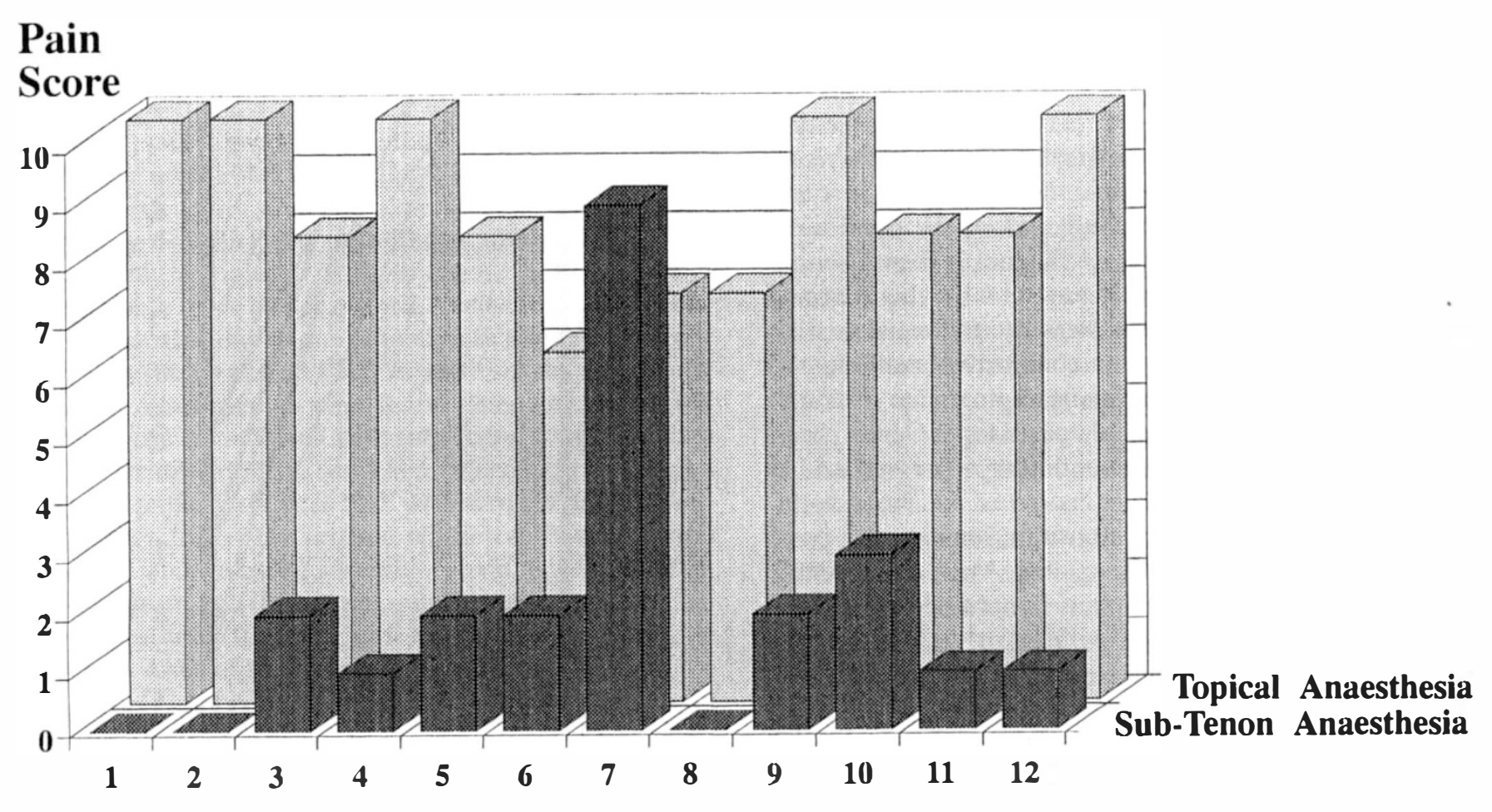

Patient Number

Fig. 2. Pain score data linking individual pain scores for PRP after topical versus sub-Tenon anaesthesia. 
random group of patients. It may be that any alternative local anaesthetic technique to that used previously may be graded as 'better' and any 'extra' anaesthesia offered may have a powerful placebo effect. For this reason bias against topical amethocaine $1 \%$ alone may be present. Within these limitations the study was designed, however, to select these 'worse case' patients for sub-Tenon anaesthesia. Toleration and completion of a PRP episode may itself be an outcome measure of success. PRP after subTenon administration was well tolerated by 11 of the 12 patients and the median pain score of 1.5 for both the administration of the block and the PRP is very low. The pain score results for the giving of sub-Tenon irrigation (median 1.5, mean 1.9) compare with previously reported scores (median 1.0, mean 1.0) for a cohort of patients undergoing cataract extraction using the same sub-Tenon technique. $^{5}$

The one patient who did not have sufficient anaesthesia by the sub-Tenon block requested termination of the PRP treatment session. The patient did not wish to continue PRP treatment at that session and therefore no 'top-up' of further anaesthetic solution was administered. An advantage of the sub-Tenon technique over injectable methods is that once the track beneath Tenon's capsule has been created, it is relatively easy to deliver further solution.

Two patients had significant pain 4 hours after treatment, providing further evidence that sub-Tenon anaesthesia had an anaesthetic effect. This pain experienced after 4 hours was a deep, aching discomfort and was not associated with the small incision in the conjunctiva since it was not associated with blinking and resolved later. To prevent post-treatment pain a longer-acting local anaesthetic combination may be used, such as a 50:50 mixture of $2 \%$ lignocaine with $0.5 \%$ bupivacaine. This is the combination used for cataract surgery involving the one-quadrant sub-Tenon technique ${ }^{5}$ and this longer duration mixture may be more appropriate for use with PRP than lignocaine $2 \%$ alone. Hyaluronidase was not used due to difficulties with supply, and the effects of its use with subTenon delivery are unknown. Adrenaline is not added to the anaesthetic agent due to the unknown effects of application of adrenaline to the posterior sub-Tenon space, where vasospasm may be expected to occur.

To compare directly the effect of sub-Tenon anaesthesia with topical treatment would involve a randomised trial of sub-Tenon anaesthesia versus topical treatment for patients who had not experienced PRP previously. Many patients experience only mild discomfort with PRP and it may not be justified to perform an invasive local anaesthetic procedure routinely. The risk-benefit ratio of any local anaesthetic technique must be considered. Though blunt cannula sub-Tenon anaesthesia seeks to avoid the risks of sharp needle techniques, it remains an invasive procedure with an incision into conjunctiva and Tenon's fascia. Some subconjunctival haemorrhage occurred in all patients, though this was not problematic since all patients were advised of this, prior to administration of the block.

If a patient tolerates PRP with simple topical anaesthesia then this is our method of choice, though if pain is experienced, further anaesthesia should be administered. If a single quadrant is to be treated, subconjunctival anaesthesia has been reported to be effective, ${ }^{1}$ though the onequadrant sub-Tenon technique offers an alternative. Use of plain lignocaine $2 \%$ can deliver anaesthesia for the PRP procedure but may provide insufficient post-treatment anaesthesia, and use of a longer-acting agent such as bupivacaine $0.5 \%$ should be considered. If PRP is required and intolerance of laser application requires additional anaesthesia, then the one-quadrant sub-Tenon technique can provide effective anaesthesia with the benefit of seeking to avoid the risks of sharp needle techniques.

Key words: Local anaesthesia, One-quadrant sub-Tenon, Panretinal photocoagulation.

\section{REFERENCES}

1. Moses KC, Norburg JW. Anaesthesia for retinal photocoagulation. Ophthalmic Surg 1990;21:156.

2. Hay A, Flynn HW Jr, Hoffman JI, Rivera AH. Needle penetration of the globe during retrobulbar and peribulbar injections. Ophthalmology 1991;98:1017-24.

3. Smith JL. Retrobulbar bupivacaine can cause respiratory arrest. Ann Ophthalmol 1982;14:1005-6.

4. Friedberg MA, Palmer RM. A new technique of local anaesthesia for panretinal photocoagulation. Ophthalmic Surg 1991;22:619-21.

5. Stevens JD. A new local anaesthesia technique for cataract extraction by one quadrant sub-Tenon's infiltration. Br J Ophthalmol 1992;76:670-4.

6. Mein CE, Woodcock MG. Local anaesthesia for vitreoretinal surgery. Retina 1990;10:47-9.

7. Stevens JD, Giubilei M, Lanigan LP, Hykin P. Sub-Tenon, retrobulbar, and peribulbar local anaesthesia: the effect upon intraocular pressure. Eur J Implant Ref Surg 1993;5:25-8.

8. Stevens JD. Curved, sub-Tenon cannula for local anaesthesia. Ophthalmic Surg 1993;24:121-2.

9. Volk Inc., 7893 Enterprise Drive, Mentor, OH 44060, USA. 